\title{
Search for charmless decays of $B$ hadrons in hadronic and radiative modes using the DELPHI detector at LEP
}

\author{
R. Nikolaidou et al
}

\begin{abstract}
Charmless hadronic decay of beauty particles can proceed through both tree level diagrams involving $\mathrm{b} \rightarrow \mathrm{u}$ transitions and penguin processes. Radiative $(\mathrm{b} \rightarrow s \gamma)$ decays are pure penguin processes. These decays are of special interest as a test of the loop structure of the Standard Model and in connection with the measurement of $\mathrm{CP}$ violation in the $\mathrm{B}$ sector. A search for charmless hadronic and radiative decays of $\mathrm{B}$ mesons has been performed with the DELPHI detector at the LEP collider. The high tracking resolution, obtained with the silicon microvertex detector, provides efficient $b$-tagging and rejection of combinatorial background. In addition, the particle identification capabilities of the DELPHI Ring Imgaging Cherenkov detector allows to separate channels with a kaon from the pure multi-pion final states.
\end{abstract}

Contribution to RICH'95, International Workshop on RICH Counters, Uppsala 12-16 June 1995 



\title{
SEARCH FOR CHARMLESS DECAYS OF B HADRONS IN HADRONIC AND RADIATIVE MODES USING THE DELPHI DETECTOR AT LEP
}

\author{
M. Battaglia ${ }^{\text {a }}$, S. Katsanevas b , D. Liko ${ }^{\text {c }}$ and R. Nikolaidou ${ }^{b *}$ \\ ${ }^{a}$ Research Institute for High Energy Physics, SEFT,Siltavuorenpenger 20c, SF-00170, Helsinki, Finland \\ ${ }^{\mathrm{b}}$ Physics Laboratory, University of Athens, Solonos 104, GR-10680, Athens, Greece \\ c Institut für Hochenergiephysik Österreichische Akademie der Wissenschaften, Nikolsdorfergasse 18, \\ A-1050, Vienna, Austria
}

Charmless hadronic decay of beauty particles can proceed through both tree level diagrams involving $\mathrm{b} \rightarrow$ $\mathrm{u}$ transitions and penguin processes. Radiative $(\mathrm{b} \rightarrow \mathrm{s} \gamma)$ decays are pure penguin processes. These decays are of special interest as a test of the loop structure of the Standard Model and in connection with the measurement of $\mathrm{CP}$ violation in the $\mathrm{B}$ sector. A search for charmless hadronic and radiative decays of $\mathrm{B}$ mesons has been performed with the DELPHI detector at the LEP collider. The high tracking resolution, obtained with the silicon microvertex detector, provides efficient b-tagging and rejection of combinatorial background. In addition, the particle identification capabilities of the DELPHI Ring Imaging Cherenkov detector allows to separate channels with a kaon from the pure multi-pion final states.

\section{Introduction}

During recent years various measurements of decay rates have been performed at colliders at the $\mathrm{b} \overline{\mathrm{b}}$-threshold. Evidence for charmless decays has been reported first by the CLEO Collaboration who observed signals in both the two-body hadronic $\mathrm{B} \rightarrow \pi(\mathrm{K}) \pi \quad$ [1] and radiative decay $\mathrm{B} \rightarrow \mathrm{K}^{*} \gamma$ [2] modes. Since the expected branching ratios for several charmless decay modes of $\mathrm{B}$ hadron are in the $10^{-5}$ range, they are becoming accessible with the present statistics by experiments at the LEP collider. There are several advantages in studying these decays at LEP. In contrast to experiments at lower center of mass energy, all $\mathrm{B}$ particle species are produced including $\mathrm{B}_{s}$ mesons and $\mathrm{B}$ baryons. The large boost of the $b$ hadrons combined with the precision measurement capability of silicon vertex detectors provides the possibility to reconstruct secondary decay vertices. Furthermore, particle identification is important for the tagging of specific final states with charged kaons and protons, allowing unambiguous determination of the decay modes. In DELPHI [3] the silicon microvertex

\footnotetext{
${ }^{*}$ Corresponding author
}

detector allows the reconstruction of decay distances in space and the Ring Imaging Cherenkov detector (RICH) [4] provides particle identification for charged tracks over a large kinematical region. The RICH identification is of special interest in this search since final charmless states containing kaons receive contributions from both tree level and penguin diagrams while those with only multi-pion states are mainly due to the tree level $\mathrm{b} \rightarrow \mathrm{u}$ decay.

\section{Hadronic decay modes}

A search for decays of B hadrons has been performed in $n$-prong final states (where $n=2,3,4$ ) containing charged kaons and protons [5]. The data sample that was used corresponds to 1.6 million hadronic $\mathrm{Z}^{0}$ decays from the 1991-1993 data taking periods. The analysis was based on the reconstruction of detached decay vertices where further kinematical cuts were applied in order to enrich the selected track sample in B decay products.

Rejection of the background coming from $\mathrm{b} \rightarrow$ $c$ decays was needed in the multi-prong analysis and was done by the identification of possi- 
ble charm states among the particles attached to the secondary vertex. The individual final states were classified using particle identification with the RICH and reconstruction of intermediate resonances. Simulation studies showed that most of the kaons coming from $\mathrm{B}$ decays have momenta in the range of coverage of the gas radiator of the RICH, which separates $(e, \mu, \pi)$ from $(K, p)$ above $3.5 \mathrm{GeV} / \mathrm{c}$ and provides exclusive kaonproton identification from $9 \mathrm{GeV} / \mathrm{c}$ to $20 \mathrm{GeV} / \mathrm{c}$. The identification algorithm used in this analysis was tuned in order to optimize its efficiency. For example, for particles tagged as kaons the efficiency was of the order of $80 \%$ independant of the particle momentum, and the rejection factor against misidentified pions was between 5 and 8 depending on the momentum.

By summing all modes in the two-body decay channel, evidence was obtained for charmless hadronic decays. Three events were found in the data in the B mass region with an estimated background of $0.29 \pm 0.09$ events. The probability that a fluctuation of the background is giving three or more events was computed to be $6 \times 10^{-3}$.

One of the two-body candidate decays, in the mode $\mathrm{B}_{\mathrm{u}}^{-} \rightarrow \mathrm{K}^{*}(892)^{0} \pi^{-}$, illustrates the power of RICH for particle identification. A display of the vertex region for this candidate is shown in Figure 1. The RICH identification properties as well as the $\mathrm{dE} / \mathrm{dx}$ data are shown in Figure 2. The RICH allowed for the unambiguous identification of the three particles.

The invariant mass distribution for the sum of all two-body decay channels is given in Figure 3 . One observes a good agreement between real data and simulated events, showing that data and Monte Carlo were consistent after application of all the cuts.

In three and four body modes no significant excess was observed due to the larger combinatorial background. Upper limits for several decay modes have been calculated. The results of the search are summarized in Table 1.

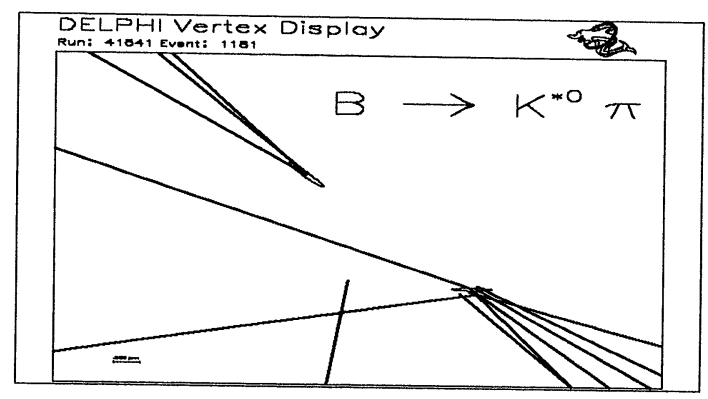

Figure 1. A magnified view of the vertex region for the candidate $B \rightarrow K^{*}(892) \pi$. In the left corner, one can see the three charged tracks coming from B decay. The primary and secondary vertices are indicated by error ellipses corresponding to $1.5 \sigma$ regions.
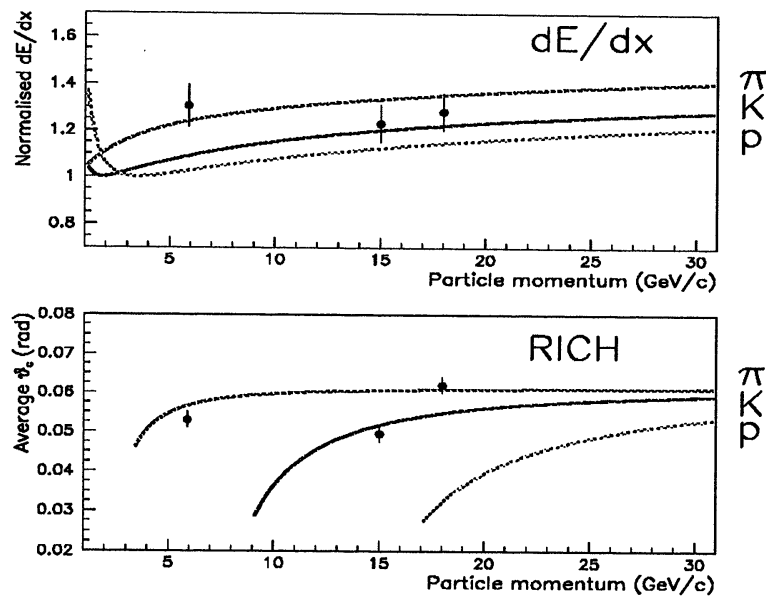

Figure 2. Particle identification with the DELPHI dE/dx and RICH systems for the same candidate as in Figure 1. The curves give the expected values for $\pi, \mathrm{K}$ and $\mathrm{p}$, as a function of momentum. The data points correspond to the 3 particles $\mathrm{B} \rightarrow \mathrm{K}^{*}(892) \pi$ with the $\mathrm{K}^{*} \rightarrow \mathrm{K}^{-} \pi^{+}$. 


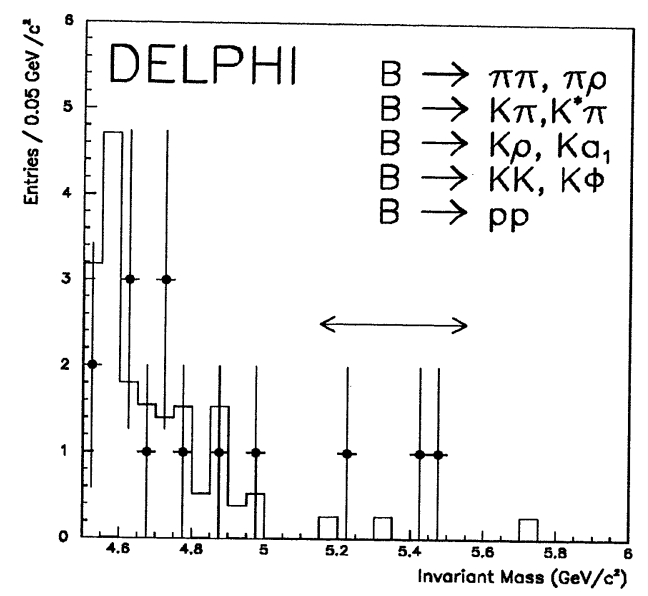

Figure 3. Invariant mass distribution for all twobody B decays. Real data are indicated by dots and simulated data by the histogram. The arrow indicates the $\mathrm{B}$ mass region.

Table 1

Summary of the upper limits (UL) at $90 \%$ confidence level. The efficiency $\epsilon$ refers to the studied charged particle decay mode.

\begin{tabular}{|l|c|c|c|c|}
\hline Channel & Data & Bkg. & $\epsilon[\%]$ & UL [Br $\left.\times 10^{5}\right]$ \\
\hline $\mathrm{B}_{\mathrm{d}}^{0} \rightarrow \pi^{+} \pi^{-}$ & 0 & .04 & 14 & 5.5 \\
$\mathrm{~B}_{\mathrm{d}, \mathrm{s}}^{0} \rightarrow \mathrm{K}^{+} \pi^{-}$ & 1 & .02 & 11 & 9 \\
$\mathrm{~B}_{\mathrm{d}, \mathrm{s}}^{0} \rightarrow \mathrm{K}^{+} \mathrm{K}^{-}$ & 0 & .002 & 5 & 12 \\
$\mathrm{~B}_{\mathrm{d}}^{0} \rightarrow \mathrm{p} \overline{\mathrm{p}}$ & 0 & .001 & 4 & 35 \\
$\mathrm{~B}_{\mathrm{u}}^{-} \rightarrow \rho^{0} \pi^{-}$ & 1 & .04 & 5 & 26 \\
$\mathrm{~B}_{\mathrm{u}}^{-} \rightarrow \mathrm{K}^{* 0} \pi^{-}$ & 1 & .02 & 4 & 48 \\
$\mathrm{~B}_{\mathrm{u}}^{-} \rightarrow \mathrm{K}^{-} \rho^{0}$ & 0 & .08 & 4 & 19 \\
$\mathrm{~B}_{\mathrm{u}}^{-} \rightarrow \mathrm{K}^{-} \Phi$ & 0 & .005 & 3.5 & 44 \\
$\mathrm{~B}_{\mathrm{d}, \mathrm{s}}^{0} \rightarrow \mathrm{K}^{+} \mathrm{a}_{1}^{-}$ & 0 & .08 & 3 & 39 \\
\hline $\mathrm{B}_{\mathrm{u}}^{-} \rightarrow \pi^{+} \pi^{-} \pi^{-}$ & 1 & 0.8 & 5 & 22 \\
$\mathrm{~B}_{\mathrm{u}}^{-} \rightarrow \mathrm{K}^{-} \pi^{+} \pi^{-}$ & 2 & 0.7 & 4 & 40 \\
$\mathrm{~B}_{\mathrm{u}}^{-} \rightarrow \mathrm{K}^{+} \mathrm{K}^{-} \mathrm{K}^{-}$ & 0 & 0.01 & 2.5 & 31 \\
$\mathrm{~B}_{\mathrm{u}}^{-} \rightarrow \mathrm{p} \overline{\mathrm{p}} \pi^{-}$ & 0 & 0.02 & 2.8 & 50 \\
\hline $\mathrm{B}_{\mathrm{d}}^{0} \rightarrow \pi^{+} \pi^{+} \pi^{-} \pi^{-}$ & 1 & 2.1 & 3.5 & 28 \\
$\mathrm{~B}_{\mathrm{d}, \mathrm{s}}^{0} \rightarrow \mathrm{K}^{+} \pi^{+} \pi^{-} \pi^{-}$ & 0 & 1.9 & 2.8 & 21 \\
$\mathrm{~B}_{\mathrm{d}}^{0} \rightarrow \mathrm{p} \overline{\mathrm{p}} \pi^{-} \pi^{-}$ & 0 & 0.06 & 1.4 & 95 \\
\hline
\end{tabular}

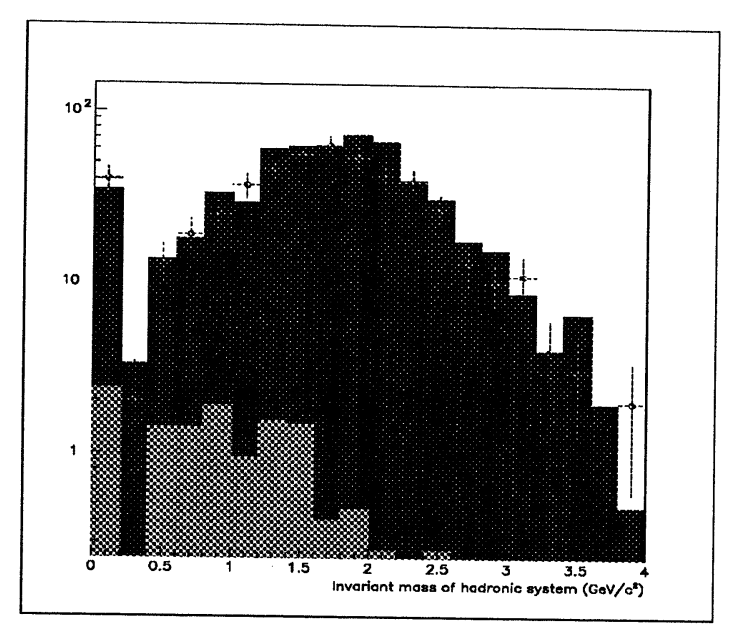

Figure 4. Invariant mass of hadronic system for data (indicated by dots) Monte Carlo events (indicated by dark grey area) and simulated $b \rightarrow$ s $\gamma$ events (indicated by the light grey area)

\section{Radiative decay modes}

An inclusive search for radiative decays of $B$ hadrons was performed on a data sample corresponding to 1.3 million hadronic $\mathrm{Z}^{0}$ decays from the 1994 data taking period. Hadronic events that had a highly energetic photon were selected for this analysis. The hadronic system accompanying the photon was reconstructed by forming a decay vertex from compatible charged tracks, $\mathrm{K}_{\mathrm{s}}^{0}$ and $\pi^{0}$ candidates. As in several cases this hadronic system was only partially reconstructed, the energy of the B hadron was estimated by correcting the visible energy with the ratio of the visible mass to that of the $\mathrm{B}$ hadron. This technique gave an energy resolution of $6.5 \%$ for the signal events. The background coming from $\mathrm{b} \rightarrow \mathrm{c}$ decays was suppressed by selecting events with a mass of the hadronic system less than $1.7 \mathrm{GeV} / \mathrm{c}^{2}$ (Figure 4).

An inclusive model [6] was used in a simulated signal sample in order to study the photon spectrum in the $\mathrm{B}$ hadron rest frame. The energy res- 
olution of the photon in the $\mathrm{B}$ hadron rest frame was found to be better than $10 \%$ (see Figure $5 \mathrm{a}$ )
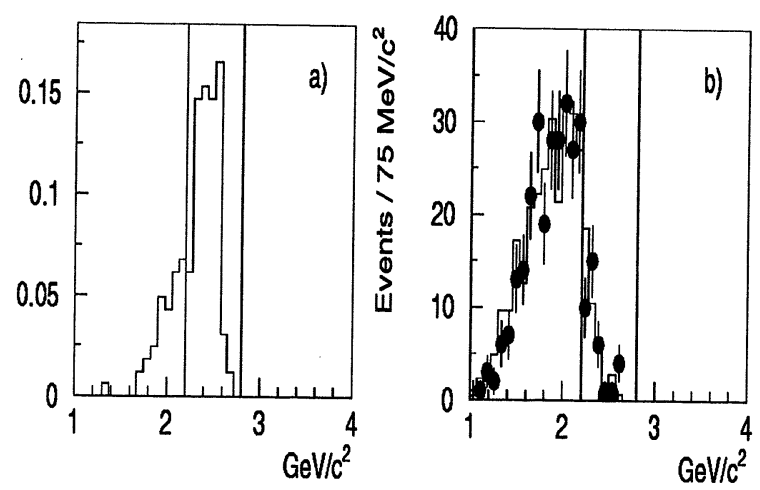

Figure 5. Photon energy spectrum in the $B$ hadron rest frame: a) expected Monte Carlo $\mathrm{b} \rightarrow \mathrm{s} \gamma$ shape smeared by the experimental resolution; b) data (dots) versus background from simulation (histogram). The vertical lines indicate the region used to obtain the upper limit.

The photon spectrum in the B rest frame for the data and simulated background events is shown in Figure 5b. Candidate $\mathrm{b} \rightarrow \mathrm{s} \gamma$ events were selected in the region between 2.2 and 2.8 $\mathrm{GeV} / \mathrm{c}^{2}$. Simulation studies showed that this region contains $60 \%$ of the reconstructed signal. The total efficiency for the reconstruction of $\mathrm{B}$ hadrons in these decays was $4 \%$. In the region of interest (see Figure 5b) 40 events were found in the data with a predicted background of 43 events. As a preliminary result this gave an upper limit at $90 \%$ confidence level for the branching ratio of

$\mathrm{BR}(\mathrm{b} \rightarrow \mathrm{s} \gamma)<9 \times 10^{-4}$

Studies for the background were done by using simulated samples of $Z^{0} \rightarrow q \bar{q}$ and $b \bar{b}$ events, which did not contain $\mathrm{b} \rightarrow \mathrm{s} \gamma$ transitions. It was found that the dominant source of background was due to prompt $\pi^{0}$ production in B decays.
This indicates that the limit can be improved by a refined photon candidate selection.

When more stringent selection criteria were used, less candidates were selected. Two events had an invariant mass in the $\mathrm{B}$ region, indicating fully reconstructed final states. One was coming from the decay of a $\mathrm{B} \rightarrow \mathrm{K}^{*} \gamma$ with the kaon identified by the RICH. Also here the RICH gives unambiguous identification of the charged kaon.

\section{Conclusions}

Decays of B hadrons into charmless hadronic modes were investigated with the DELPHI detector at LEP, using 1.6 million hadronic $\mathrm{Z}^{0}$ decays. Evidence for these decays was found in two-body modes where the particle identification capability of the RICH detector was used to tag the final states with protons, kaons and pions. No excess has been found in the higher multiplicity modes. Upper limits for several decay modes have been calculated.

In the radiative decay modes of $\mathrm{B}$ hadrons an inclusive search has been performed and an upper limit for the branching ratio of $\mathrm{b} \rightarrow \mathrm{s} \gamma$ has been given. When all data will be analyzed, the limit will be improved and evidence for this rare decay might be obtained.

\section{REFERENCES}

1 M. Battle et al. (CLEO), Phys. Rev. Lett. 71 (1993) 3922.

2 R. Ammar et al. (CLEO), Phys. Rev. Lett. 71 (1993) 674.

3 P. Aarnio et al., Nucl. Instr. Meth. A 303 (1991) 233.

4 E. Anassontzis et al., Nucl. Instr. Meth. A 323 (1992) 351.

5 P. Abreu et al. (DELPHI Collaboration), Search for charmless hadronic $B$ decays CERN-PPE 95-91 Subm. to Phys. Lett.

6 A. Ali and P. Greub, Phys. Lett. B 259 (1991) 182. 